\title{
Responsabilidad social, más allá del pregrado: Consideraciones para la formación ciudadana y la gestión estratégica
}

\author{
Social Responsibility, beyond the undergraduate level: \\ Considerations for citizen training and strategic management \\ Verónica Rubio Aguilar \\ Universidad Santo Tomás, Viña del Mar, Chile.凶 vrubioa@santotomas.cl \\ [orcid.org/0000-0002-8965-1461]
}

\begin{abstract}
RESUMEN
La Responsabilidad Social (RS) no puede reducirse al aprendizaje de competencias en estudiantes, hace falta que se enraíce en académicas y académicos para considerarla como eje estratégico de toda gestión universitaria que contribuya a la formación ciudadana, tributando a la transformación de sociedades más inclusivas, democráticas e interculturales, especialmente considerando el presente escenario, interpelado por demandas sociales y crisis sanitaria. Este artículo sistematiza los resultados de una investigación cualitativa orientada a analizar cómo significan la RS, las y los académicos de una universidad chilena y su homóloga en Mozambique, después de su formación de postgrado. Los resultados del análisis interpretativo evidencian que alcanzaron un conocimiento actualizado de RS, rompiendo con el paradigma asistencialista que detentaban, logrando incorporarla en los ejes de docencia, gestión, investigación y extensión académica desde una perspectiva integral y no residual, identificando críticamente algunos desafíos pendientes de la educación superior chilena para favorecer una ciudadanía socialmente responsable.
\end{abstract}

PALABRAS CLAVE: Responsabilidad social, formación ciudadana, gestión estratégica, educación superior.

\section{ABSTRACT}

Social Responsibility (SR) cannot be reduced to the learning of competencies in students, it must be rooted in academics to be considered as a strategic axis of all university management that contributes to the formation of citizens, contributing to the transformation of more inclusive, democratic and intercultural societies, especially considering the present scenario, challenged by social demands and health crisis. This article systematizes the results of a 
qualitative research oriented to analyze how SR means, the academics of a Chilean university and its counterpart in Mozambique, after their postgraduate training. The results of the interpretative analysis show that they achieved an updated knowledge of SR, breaking with the welfare paradigm that they held, managing to incorporate it into the axes of teaching, management, research and academic extension from a comprehensive and non-residual perspective, critically identifying some pending challenges of Chilean higher education to promote socially responsible citizenship.

KEY WORDS: Social responsibility, citizenship training, strategic management, higher education.

\section{INTRODUCCIÓN}

Es reconocible para quienes habitamos América Latina y El Caribe, el rol fundamental que se le atribuye a la educación superior como medio, instrumento, meta o utopía, para alcanzar el progreso de las sociedades, por medio de la formación profesional de las nuevas generaciones. La superación de la pobreza, las condiciones de desigualdad e inequidad, la inclusión, son urgencias en nuestro continente tan legítimas de superar, como la aspiración de acceder, mantenerse y egresar exitosamente de una carrera profesional o técnica, para alcanzar el tan anhelado ingreso al mercado laboral. La educación superior debe entonces aportar a la formación de profesionales, ciudadanos y ciudadanas, que velen por la equidad social, el mejoramiento de la distribución del ingreso, la inclusión en todas sus expresiones y orienten su accionar al desarrollo sustentable, en un contexto de acelerada globalización y tecnologización, disminuyendo brechas y cruzando puentes.

La universidad es uno de los actores invitados a identificar y aportar soluciones a los problemas de interés público que hoy nos agobian, a través de estrategias e iniciativas ciudadanas desde la transparencia, pluralidad y sustentabilidad, en un marco de ética cívica y dialógica, donde aportemos a nuevas políticas públicas verdaderamente participativas, desde la cooperación multidisciplinar (Rubio, 2019, p. 10).

Por ello se le otorga a la educación superior un alto sentido de Responsabilidad Social (RS), que ya en 2009 ratificó la Conferencia Mundial sobre Educación Superior en su sede de la UNESCO en París, donde declara que nunca en la historia de la humanidad el bienestar de las naciones dependió de una manera tan directa de la calidad de sus sistemas educativos e Instituciones de Educación Superior, por tanto, debe ser un tema de responsabilidad de todos los gobiernos, puesto que como bien público juega un rol importante en la construcción de las naciones (UNESCO, 2009). Posteriormente varios otros acuerdos internacionales reafirman esta declaración, el último de ellos es el llevado a cabo en la III Conferencia Regional de Educación Superior, celebrada en junio de 2018, en la Universidad Nacional de Córdoba, Argentina, con motivo de la conmemoración de los cien 
años de la primera declaración en 1918, donde se señala que la responsabilidad social de las instituciones de educación superior exige una nueva relación con la sociedad y postula una transformación innovadora de la educación superior (CRES, 2018).

Existen distintas acepciones de Responsabilidad Social que se vinculan a diversos ámbitos y niveles de acción. En el caso de la educación superior, específicamente de las universidades, resulta muy útil comprenderla como Responsabilidad Social Universitaria (RSU). Es decir, como

la capacidad que tiene la universidad, de difundir y poner en práctica un conjunto de principios y valores generales y específicos, por medio de cuatro procesos considerados claves: la gestión, la docencia, la investigación y la extensión universitaria, respondiendo socialmente así, ante la propia comunidad universitaria y el país donde está inserta (Proyecto Universidad Construye País, 2006, p. 35).

Esta definición surge en Chile, país considerado pionero en Latinoamérica en inserción de la RS en educación superior, que dio lugar a una iniciativa que apoyada por la Corporación PARTICIPA y la Fundación AVINA, reunió en sus inicios a once universidades chilenas, dando lugar en el año 2001, al Proyecto Universidad Construye País, inspirado en la Declaración de Glion, Suiza, y en la Conferencia Mundial sobre Educación Superior de Unesco, ambas efectuadas en 1998. El propósito de este proyecto fue expandir el concepto y la práctica de la responsabilidad social universitaria (Proyecto Universidad Construye País, 2002). Es así como las universidades tanto públicas como privadas, inicialmente se caracterizaron por la tendencia a desarrollarla más en docencia (formación de pregrado en asignaturas complementarias al currículum) y extensión universitaria (estrategias de aprendizaje servicio), pero menos en investigación (en temáticas de RS o en investigaciones socialmente responsables) y en "gestión (ética e inteligente) de los impactos organizacionales" (Vallaeys, 2009, p. 28). Esto ha significado que la implementación de la RS en las universidades, en sus orígenes en Chile y en Latinoamérica a inicios del siglo XXI, fuese más bien externa (extensión académica) en lugar de interna (gestión organizacional), centrada en los estudiantes (formación de pregrado) más que en otras y otros actores que también conforman los sistemas educativos, como lo son directivos, académicos y funcionarios. Esto puede corroborarse cuando se identifica que las investigaciones en RS efectuadas en Chile, entre 2002 a 2012, reflejan una tendencia por la preferencia de estudios de corte cuantitativo orientados a establecer diagnósticos "que abarcan un número relevante de sujetos de estudio y cuyos resultados pueden operar como predictores de conductas, actitudes y variables asociadas a la RS, preferentemente en estudiantes, entendiendo ésta como un fenómeno individual" (Rubio, 2013, p. 21). Posteriormente, la RS además de ser gestionada e investigada en los estudiantes, ha considerado a otros actores, como lo son las instituciones, los gobiernos y la ciudadanía (Navarro et al. 2017), ampliando la concepción individual a una organizacional, sin perder de vista sus énfasis distintivos y necesarios de gestionar de manera sistémica y relacional. No obstante, el foco puesto en otros actores diversos a los estudiantes sigue siendo menor. En síntesis, la expansión de la RS en el sistema educativo en Chile ha sido sostenida y creciente 
en el tiempo, especialmente a través del reconocimiento del Aprendizaje Servicio como “metodología que potencia el aprendizaje experiencial, caracterizado... por...: detección de una necesidad social o comunitaria genuina, relación entre objetivos curriculares y objetivos de servicio, asignación de espacios estructurados para la reflexión y participación protagónica de los estudiantes" (Pizarro \& Hasbún, 2019, p. 37); "como oportunidad para repensar la relación que la academia ha tenido con sus contextos sociales más próximos, ....como.... actores sociales que conjunta y dialécticamente van transformando y transformándose en un contexto histórico-social y político concreto" (Vivero, Molina \& Standen, 2020, p. 135). Observándose distintas etapas de institucionalización, desde la creación de masa crítica; construcción de calidad, hasta llegar a la institucionalización sustentable (Pizarro \& Hasbún, 2019, p. 35), donde si bien participan docentes y en ocasiones existe incorporación en los planes de desarrollo estratégico de algunas universidades, el énfasis sigue estando puesto en la formación de los estudiantes.

El estudio que aquí se presenta se llevó a cabo en una universidad cuya misión se orientaba hasta 2018 a formar personas con un nivel de preparación profesional, valórica y actitudinal que les permita desempeñarse con integridad, eficiencia, creatividad y responsabilidad social, contribuyendo al desarrollo de su familia y comunidad, aportando a la cultura y a la generación de conocimiento. Para esto, ha optado por variadas estrategias para promover la RS, siendo una de ellas el fortalecimiento de su cuerpo académico y directivo. Para ello, dicta el programa de Magíster en Gestión en Educación Superior buscando que sus graduados y graduadas sean capaces de evaluar críticamente las tendencias de política y gobierno en Chile y Latinoamérica, además de aplicar herramientas de gestión estratégica en educación superior. En el decreto que lo aprueba se fundamenta que este programa de postgrado tiene una visión comprehensiva de la educación superior, abarcando universidades, institutos profesionales y centros de formación técnica; enfatiza una perspectiva analítica internacional y comparada de la política y gestión en educación superior; profundiza en sus fundamentos socio-políticos y técnicos, proveyendo una base teórico-conceptual y un andamiaje instrumental aplicable en todas las instituciones del sector. En síntesis, busca que sus graduados y graduadas evalúen críticamente la situación y tendencias de política y gobierno en educación superior en Chile y países relevantes y apliquen herramientas de gestión estratégica y control de procesos orientado a resultados clave de las instituciones de educación superior.

En el segundo semestre de formación de este programa se encuentra la asignatura Vinculación con el Medio (VCM) y Responsabilidad Social (RS) en Educación Superior, cuyo propósito es introducir a los y las estudiantes en la conceptualización y análisis de las tendencias en gestión de la vinculación con el medio, con énfasis en la Responsabilidad Social Universitaria (RSU), comprendiendo la importancia de una gestión socialmente responsable, examinando algunas de las experiencias exitosas en este campo y entregando herramientas que favorezcan la formulación de proyectos y acciones de vinculación con el medio con indicadores de RS, con el fin de alcanzar las siguientes capacidades: reconocer diferentes sustentos teóricos y conceptuales para comprender la importancia de la RS en la gestión de los distintos sistemas de educación superior; valorarla como eje central 
en la gestión de los diferentes sistemas que conforman la educación superior en Chile, especialmente en lo que se refiere a vinculación con el medio; desarrollar habilidades que favorezcan la formulación y puesta en marcha de proyectos y acciones de vinculación con el medio con estándares de RS.

Este artículo sistematiza los principales resultados de una investigación cualitativa orientada a analizar cómo significan la RS, las y los estudiantes de la cátedra VCM y RS, dictada en los años 2014, 2016 y 2017, desde los testimonios de quienes la cursaron: 41 académicos y directivos de un sistema educativo conformado por tres plataformas de educación superior: centro de formación técnica, instituto profesional y universidad. Según esto, fue posible identificar diferentes sentidos, creencias, valores y prácticas asociadas a la RS, de acuerdo con los significados que le atribuye este grupo particular, por cuanto las personas comprenden el mundo mediante la interacción con otros, y con sus entornos cercano y remoto. De esta forma los significados traspasan el ámbito de lo privado y se vuelven públicos, cuando las personas participan de grupos y culturas determinadas.

El foco de interés estuvo puesto en conocer el impacto que la formación en RS puede tener en quienes están a cargo de los procesos de aprendizaje de los nuevos profesionales, es decir, las y los académicos. Siendo un dato relevante, desde el que se sostiene este estudio, que las investigaciones de estos actores sociales aún son escasas, existiendo a la fecha poca información que se centre en ellos. Por lo tanto, la aproximación al campo de estudio se hizo desde un eje distinto a la indagación de la formación de este valor y buena práctica en las y los estudiantes, en el convencimiento que la RS es una variable urgente de incorporar en la planificación estratégica de los sistemas educativos contemporáneos, lo que puede intencionarse si se forma en quienes los lideran, sus académicos y no sólo en quienes los integran como estudiantes. De allí que esta investigación se orientó a analizar ¿cómo significan la RS, las y los académicos de una institución de educación superior chilena y su homóloga en Mozambique, después de su formación de postgrado?, para conocer cómo la comprendieron e incorporaron en la gestión de diversas unidades académicas.

\section{CONSIDERACIONES TEÓRICAS}

A continuación, se presentan los sustentos teóricos que fundamentan esta investigación, los que dicen relación con: nociones de RS; impactos de la gestión universitaria, una cuestión de RS; responsabilidad desde la filosofía del lenguaje y significados de RS.

\section{Nociones de Responsabilidad Social}

Todo el mundo tiene más o menos claro el concepto de responsabilidad que deriva del latín respondeo que significa dar cuenta, "sin embargo, cuando se conjuga con las palabras: social, social empresarial, social ética, social universitaria, empiezan las confusiones" (Rubio, 2012, p. 31). Lo mismo ocurre cuando se habla de responsabilidad social empresarial y 
responsabilidad social universitaria. Lo cierto es que la RS, ya sea entendida como valor, actitud o conducta, es hoy reconocida como un eje fundamental de gestión que toda organización debe incorporar de manera transversal en su planificación estratégica. Esto lo ratifican Navarro y colaboradores (2017) cuando en su investigación que analiza el contexto actual de la RS en tres países de Latinoamérica: Perú, Argentina y Chile, plantean que es necesario conocer las razones y propósitos de incorporarla en la formación de personas, en la administración pública y gobierno, lo que según las autoras requiere comprenderla como valor, comportamiento, educación para la RS y eje estratégico de gestión organizacional y, por tanto, universitario. Una breve revisión de algunas acepciones muestra cómo puede ser desarrollada desde una perspectiva individual a una corporativa y hasta societal.

Por ello las Universidades no podían quedarse alejadas de la reflexión sobre la RS, que ya muchas empresas han desarrollado durante los últimos años, no sólo porque ellas también son organizaciones, sino porque además les corresponde formar a los futuros profesionales que laborarán en las organizaciones y empresas, a los futuros ciudadanos que tendrán que promover democráticamente los derechos humanos y a quienes tendrán a su cargo el bien común de este ya reconocido mundo globalizado (Rubio, 2012, p. 33).

Una perspectiva que enfatiza en lo individual es la que propone Sáez cuando la comprende como la "capacidad y obligación de responder ante la sociedad como un todo, por acciones u omisiones y se ejerce, cuando corresponde, desde alguna persona hacia todas las otras” (Sáez, 2001, p. 50). En complemento, Urzúa (2001) pone el acento en la articulación de las personas con la sociedad cuando define la RS como

la orientación de las actividades individuales y colectivas en un sentido que permita a todos, igualdad de oportunidades para desarrollar sus capacidades, suprimiendo y apoyando la eliminación de los obstáculos estructurales de carácter económico y social, así como los culturales y políticos que afectan o impiden ese desarrollo (p. 55).

La eliminación de obstáculos estructurales: sociales, económicos, culturales y políticos requiere de capacidades individuales y disposiciones organizacionales donde la educación superior juega un papel relevante en el logro del rol social que le cabe, más allá de la formación profesional y especializada que pueda otorgar a las nuevas generaciones. Es por ello como lo plantea Bacigalupo (2006)

que hoy se habla de RS universitaria en lugar del antiguo concepto de proyección social puesto que no se trata de que la universidad se proyecte con lo que tiene de sobrante a la sociedad, sino que responda a demandas sociales específicas. Esto quiere decir que a la Universidad se le está demandando una cierta injerencia política orientada al cambio de estructuras y hábitos sociales que impiden el bienestar y el desarrollo humano (p. 29). 


\section{Impactos de la gestión universitaria, una cuestión de responsabilidad social}

Uno de los conocimientos clave a que nos invita la RS, es tener en cuenta los impactos que generan nuestras acciones: en los otros, en nuestro entorno inmediato y en el medio ambiente. En este sentido la universidad también debe cuestionarse por los impactos específicos que genera al operar en su entorno, los que según Vallaeys en 2006, pueden ser de cuatro tipos:

Impacto organizacional: la universidad genera impactos en la vida de su personal (que su política de bienestar social debe de gestionar), y también contaminación en su medio ambiente (desechos, deforestación, polución atmosférica). Los riesgos de este impacto es que una universidad que mantiene una cultura organizacional burocrática, no democrática, elitista, ciega a los problemas sociales y ambientales que genera, con hábitos de despilfarro y sin política específica de selección de sus proveedores, le está trasmitiendo de manera oculta estos mensajes a sus estudiantes y a la sociedad.

Impacto educativo: la universidad tiene un impacto directo sobre la formación de los jóvenes y profesionales. Su manera de entender, interpretar e imaginar el mundo, comportarse en él y valorar ciertas cosas más que otras. Influye asimismo sobre la deontología profesional, orienta la definición de la ética profesional y de su rol social. Como riesgo se puede apreciar en este eje a aquella universidad que promueve, desde sus planes de estudios, una educación trunca, desligada de los problemas sociales, con fines instrumentales, basada en una relación asimétrica profesor/alumno, sin afán de formación integral ciudadana.

Impacto científico y epistemológico: la universidad orienta la producción del saber, influye en la definición de lo que se llama socialmente "verdad, ciencia, objetividad, racionalidad, legitimidad, utilidad”. Participa en la delimitación de los ámbitos de cada especialidad, así como en la definición de los problemas de la agenda científica. Articula la relación entre tecnociencia y sociedad. Este impacto corre riesgos cuando la universidad incentiva la fragmentación y separación de los saberes, invisibiliza los problemas inter-multi-trans-disciplinarios, promueve actitudes como el elitismo científico ("expertocracia”), desalienta el control social democrático de la ciencia, produce conocimientos socialmente irrelevantes o, al contrario, se somete a pedidos coyunturales (confusión entre investigación y consultoría).

Impacto social: la universidad tiene un impacto sobre la sociedad y su desarrollo económico, social y político. No sólo forma a los futuros líderes y profesionales de la nación, sino que es también un referente y actor social. En este aspecto el riesgo que corre la universidad es que puede reducirse a la colocación de jóvenes en el mercado laboral, desentendiéndose de la pobreza o promoviendo el elitismo y la segregación social (generando “capital antisocial”), que desvincula la formación académica de la acción social o se satisface con iniciativas de voluntariado asistencialista (Vallaeys, 2006, p. 33). 
La consideración de estos impactos colabora en la comprensión de la RSU como una estrategia de gerencia ética e inteligente de los impactos que genera la universidad en su entorno humano, social y natural como lo explica Vallaeys. Gerencia ética porque todos los potenciales afectados por la actividad de la organización deben retirar los mayores beneficios y menores daños de ella. La organización debe servir al mundo y no sólo servirse del mundo. Gerencia inteligente porque la gestión responsable de los impactos de la organización debe retornar en beneficios para la misma. Al ser socialmente responsable, la organización se desarrolla mejor en un entorno mejor (Vallaeys, 2009).

\section{Responsabilidad desde la filosofía del lenguaje}

Para comprender los antecedentes teóricos y conceptuales examinados, desde un marco de análisis más amplio, como es el de la Responsabilidad, cabe relevar la figura de dos filósofos representantes del giro lingüístico de la filosofía. El primero de ellos, Karl Otto Apel, quien desde el punto de vista de una Ética de la Responsabilidad o también denominada Ética Discursiva plantea la necesidad de una cooperación interdisciplinaria para tematizar y resolver las consecuencias no esperadas de la era de la ciencia (Apel, 1985), donde se hace imperativo avanzar de un sentido de responsabilidad individual a una corresponsabilidad solidaria, resaltando la condición dialógica de los seres humanos. Apel va más allá incluso cuando plantea textualmente que "la exigencia de mantener la vida es un derecho de todos los seres humanos y esta exigencia está garantizada en una comunidad ideal de comunicación” (Apel, 1985, p. 121). Por su parte y en complemento, Hans Jonas desde la formulación del Principio de Responsabilidad, plantea radicalmente la problemática de la responsabilidad humana frente a las consecuencias del desarrollo científico tecnológico y del ilimitado poder que han alcanzado los hombres sobre el mundo natural y social, enfatizando los peligros que implica el desarrollo actual de la técnica para la supervivencia humana (Jonas, 1995). Ante este diagnóstico critica el abandono de la responsabilidad a su dimensión puramente verbal o formal, planteando la imperiosa necesidad de analizar la problemática de la responsabilidad por la vida futura de la humanidad, formulando el siguiente imperativo ético: “actúa de tal modo, que las consecuencias de tu acción sean compatibles con la permanencia de una verdadera vida humana sobre la tierra” (Siqueira, 2001, p. 278). Los postulados de Jonas coinciden en este aspecto con los de Apel con quien comparte el diagnóstico relacionado con los peligros globales y la irreversibilidad de las consecuencias de la técnica en el mundo contemporáneo (Apel, 2007), como también la necesidad de elaborar una ética de la responsabilidad orientada al futuro, que sea capaz de superar las concepciones históricoabstractivas de las primeras éticas, como la kantiana.

En el contexto de la educación superior, como lo propone Rubio (2012), es posible trasladar los aportes de Apel y Jonas al entendimiento de las instituciones de educación superior (universidades, institutos profesionales y centros de formación técnica), como comunidad ideal de comunicación, que dialoga y se ocupa de su ambiente, transformándose en organizaciones de calidad socialmente responsables. Para ello propone, a modo 
ilustrativo, que la universidad deberá transitar en los casos más extremos de ser una unidad sólo centrada en la captación de estudiantes clientes, sin medir los impactos que genera y sin apreciar las demandas de desarrollo país que se requieren cubrir, a tensionarse e implicarse para convertirse en una universidad dialógica que responda a las demandas contextuales aportando tanto al desarrollo individual de las y los profesionales que forma, como al desarrollo social y económico de la sociedad en que se inserta.

En este diálogo, deben estar presentes todas las posiciones de enunciación como dice Preciado (2002) y todas las posibilidades de argumentación legítimamente orientadas al consenso, como recalca Apel (2007), ocupándonos porque los intereses de todos y cada uno estén representados, especialmente de quienes son considerados vulnerables y son más bien vulnerados: niños, niñas y adolescentes, mujeres, personas mayores, trabajadores, pobladores, migrantes, pueblos originarios, identidades diversas y disidentes, entre muchos otros (Rubio, 2019, p. 10).

\section{Significados de responsabilidad social}

Desde la perspectiva del construccionismo social, los significados pueden entenderse como

formas de interpretar y comprender el mundo, y la realidad social de los sujetos, incluyendo sus creencias y valores, los cuales son construidos socialmente en la interacción con otros, situados en un contexto histórico y cultural, que es exteriorizado a través de una serie de argumentaciones, discursos y premisas, que connotan las acciones de los individuos (Gergen, 2006, p. 75).

Esta propuesta teórica se arraiga epistemológicamente en el giro lingüístico de la filosofía, de allí la coherencia de utilizarla en este estudio, puesto que es distinguida como una de las concepciones postmodernas de la psicología social que elabora una crítica a la concepción representacionista del conocimiento, rechaza la noción explicativa y causal del conocimiento, junto con la noción de acumulación y progreso científico, dando paso a una comprensión relativista que propicia la comprensión de las narrativas, las relaciones y el análisis del discurso (Cañón et al. 2005, p. 240), que pone el acento en la condición lingüística y dialógica de las personas, así como lo proponen la Ética Discursiva de Apel y el Principio de Responsabilidad de Jonas. Según esto, descubrir acepciones, valores, creencias y prácticas sociales, es fundamental para aproximarnos a la comprensión de los significados que ciertos grupos atribuyen a determinados hechos o valores. En este caso particular significados de RS en académicos y directivos de educación superior. En ello, colabora la revisión de algunas premisas del construccionismo social, consideradas en este estudio: 
Los términos con los cuales se comprende el mundo son artefactos sociales, es decir, productos de intercambios entre la gente, históricamente situados. El proceso de entender no es dirigido automáticamente por la naturaleza misma de las cosas, sino que resulta de una empresa activa y cooperativa de personas en relación. De este modo los conceptos y significados de RS asumen distintas consideraciones dependiendo de las interacciones de personas en contextos históricamente situados. Por eso hoy el concepto de RS adquiere una trascendencia mayor a épocas anteriores, como también podrá variar, si las personas manejan concepciones modernas o se adhieren a una determinada corriente filosófica, ideológica y hasta confesional de RS. Todo lo que influirá en las valoraciones y creencias asociadas a la RS.

Lo que se considera conocimiento del mundo no es producto de la inducción o de la construcción de hipótesis generales, sino que está determinado por la cultura, la historia o el contexto social. De este modo las expresiones de RS pueden estar definidas de manera diversa, desde su uso social en cada institución educativa, lo que a su vez es producto de la cultura e historia que las caracteriza y las distingue de otras. Es decir, las personas entregan con sus significaciones, la visión del contexto al que pertenecen respecto de cómo aprecian y vivencian la RS.

El lenguaje es un subproducto de la interacción, cuyo principal significado se deriva del modo en que está inmerso dentro de patrones de relación (Pakman, 1997). Es decir, la importancia del lenguaje se puede comprender como un medio que permite a las personas volverse perceptibles a través de sus prácticas sociales. Sin el lenguaje, la experiencia del mundo sería un flujo invisible, sin estructura ni significado. La organización del lenguaje determina la manera cómo la experiencia y la conciencia se relacionan, en este caso mediante prácticas de y en torno a la RS. Es así, como se vuelve importante comprender el sentido de la acción social desde las personas que interactúan e intercambian visiones de mundo, lo que va a depender netamente del lenguaje.

\section{METODOLOGÍA}

Este artículo analiza testimonios de los y las profesionales que cursaron la asignatura de VCM y RS en las tres versiones en que se ha dictado, fundamentado paradigmáticamente desde el enfoque fenomenológico (Vasilachis de Gialdino et al. 2006). Por ello la metodología seleccionada fue cualitativa (Salgado, 2007), el tipo de estudio descriptivo interpretativo (Sabino, 1986) con un diseño flexible, naturalista y sin control (Mella, 2003), que permitió analizar el objeto de estudio, a partir de las expresiones y actividades de las personas investigadas (Flick, 2012).

Los datos fueron recogidos a través de entrevistas focalizadas, individuales y grupales, siguiendo lo propuesto por Flores (2009) y Canales (2006), con el fin de apreciar a través de 
los relatos: conocimientos, creencias, valores y prácticas asociadas a la RS, antes, durante y después de la asignatura. En complemento, con el objetivo de alcanzar un conocimiento más amplio, se estimó necesario en cada entrevista consultar a las personas investigadas, respecto de algunos productos derivados de la asignatura, tales como: contenidos de clases, evaluaciones, talleres en aula y trabajos elaborados por los y las estudiantes. Para asegurar la validez y fiabilidad, el guion temático fue previamente sometido a juicio de expertos y se hizo una entrevista piloto, antes de su aplicación definitiva. Cada entrevista efectuada fue grabada, previa autorización de las personas, mediante la puesta en marcha de un protocolo de consentimiento informado, con el fin de resguardar su dignidad, anonimato y confidencialidad, antes, durante y después de todo el proceso investigativo, el que fue previamente aprobado por el Comité de Ética Científico de la Universidad que efectúa este estudio. Las entrevistas fueron llevadas a cabo durante los años 2017 a 2019, aplicando una a dos entrevistas a las personas seleccionadas en sus lugares de trabajo, en dependencias de la universidad donde cursaron el programa de postgrado, incluyendo cuando fue necesario la recogida de datos mediante entrevistas escritas, previa entrega de guion temático de preguntas.

Las y los participantes de la investigación fueron seleccionados de acuerdo con el muestreo no probabilístico de tipo intencionado propuesto por Lagares y Puerto (2001) y Martínez-Salgado (2012), debido a sus posibilidades de ofrecer información profunda y detallada, sobre el interés del estudio. Para ello de acuerdo con criterios de heterogeneidad, representatividad y accesibilidad, se seleccionaron diez personas, entre los 34 a 60 años, que cumplieran con el criterio de inclusión de haber cursado y aprobado la cátedra de Vinculación con el Medio y Responsabilidad Social en Educación Superior, del Magíster en Gestión en Educación Superior. De esta forma, la muestra estuvo compuesta por cinco hombres y cinco mujeres, académicos, académicas y/o directivos o directivas a cargo de áreas de gestión en docencia, administración, finanzas o formación valórica.

El análisis de datos fue llevado a cabo mediante la técnica de contenido interpretativo elaborada por Gómez-Mendoza (2000), análisis que fue validado por un proceso de triangulación múltiple (Pérez, 2002) con las personas investigadas y con una investigadora experta en metodología cualitativa para validar que el proceso de análisis de datos fue efectuado de manera adecuada.

\section{RESULTADOS}

Previo a la revisión de los resultados principales de este estudio, corresponde conocer el perfil social de cada una de las personas entrevistadas, con el fin de colaborar en la comprensión de sus respuestas e interpretaciones, como se expone en la Tabla 1. Participantes de la Investigación.

Los resultados de la investigación se organizaron de acuerdo con la información recogida respecto de las tres capacidades a lograr en el programa de la asignatura, a saber: sustentos teóricos y conceptuales de RS, valoración de la RS como eje de gestión universitaria, desarrollo de habilidades para la formulación de proyectos de VCM con RS. 


\section{RuBio}

Tabla 1. Participantes de la investigación.

\begin{tabular}{|c|c|c|c|c|}
\hline Participante & Género & Edad & Profesión & Actividad actual \\
\hline Entrevistada 1 & Mujer & 42 años & $\begin{array}{l}\text { Profesora de } \\
\text { filosofía }\end{array}$ & $\begin{array}{l}\text { Académica y directora de unidad de RS en } \\
\text { sede de la universidad en el extranjero }\end{array}$ \\
\hline Entrevistada 2 & Mujer & 45 años & Chef & $\begin{array}{l}\text { Académica y jefe de carrera de } \\
\text { Gastronomía en centro de formación } \\
\text { técnica de zona sur }\end{array}$ \\
\hline Entrevistada 3 & Mujer & 45 años & Diseñadora & $\begin{array}{l}\text { Académica y jefe de carrera de Diseño en } \\
\text { instituto profesional de zona sur }\end{array}$ \\
\hline Entrevistada 4 & Mujer & 35 años & Nutricionista & $\begin{array}{l}\text { Académica y jefe de carrera de Nutrición y } \\
\text { Dietética en universidad de zona norte }\end{array}$ \\
\hline Entrevistada 5 & Mujer & 40 años & Abogada & $\begin{array}{l}\text { Académica y directora de carrera de } \\
\text { Derecho en universidad de zona sur }\end{array}$ \\
\hline Entrevistado 1 & Hombre & 60 años & Biólogo marino & Rector de universidad de zona sur \\
\hline Entrevistado 2 & Hombre & 34 años & $\begin{array}{l}\text { Ingeniero en } \\
\text { Informática }\end{array}$ & Académico de universidad de zona centro. \\
\hline Entrevistado 3 & Hombre & 60 años & Ingeniero Civil & Académico de universidad de zona centro \\
\hline Entrevistado 4 & Hombre & 35 años & $\begin{array}{l}\text { Profesor de } \\
\text { Religión }\end{array}$ & $\begin{array}{l}\text { Académico y director área de Formación e } \\
\text { Identidad, de centro de formación técnica, } \\
\text { instituto profesional y universidad zona } \\
\text { norte }\end{array}$ \\
\hline Entrevistado 5 & Hombre & 45 años & $\begin{array}{l}\text { Contador } \\
\text { Auditor }\end{array}$ & $\begin{array}{l}\text { Director área de Finanzas de centro de } \\
\text { formación técnica, instituto profesional y } \\
\text { universidad de zona norte }\end{array}$ \\
\hline
\end{tabular}

Fuente: Elaboración propia.

\section{Sustentos teóricos y conceptuales de Responsabilidad Social}

La primera capacidad a lograr de la asignatura se orienta a que los y las profesionales reconozcan diferentes sustentos teóricos y conceptuales para comprender la importancia de la RS en la gestión de los distintos sistemas de educación superior. En este ámbito, las personas investigadas expresan una diferenciación muy clara en el entendimiento de la RS, que refleja dos polos de un mismo continuo: conocimientos antes y después de la asignatura. De esta forma hay coincidencia en todos los relatos respecto de que transitaron desde la ausencia casi total de conocimiento conceptual, ausencia total de conocimiento teórico y manifestación de muchos mitos respecto de la RS, hasta alcanzar conceptos adecuados y actualizados de RS, identificación de sustentos teóricos asociados y comprensión adecuada de lo que es y no es RS, logrando además discriminar y clasificar la información aprehendida en distintas categorías: orígenes de la RS; conceptualizaciones básicas y conceptos asociados; diferenciaciones conceptuales; qué y que no es RS; mitos y realidades al respecto. 
En lo conceptual los entrevistados en su mayoría reconocen que, al iniciar la asignatura, disponían de significaciones de RS orientadas al asistencialismo, a la filantropía, a acciones externas y verticales de RS de las instituciones de educación superior y a conductas de carácter individual y de voluntariado no profesional, por sobre lo colectivo y promocional en las personas. Dichas significaciones no se sustentaban en un conocimiento formal y sistemático, sino que provenían de aproximaciones esporádicas a experiencias de otras personas con la temática.

Yo pensaba que la RS sólo era asistencialismo (Entrevistado 1).

No sabía que la RS podía ser interna y externa a la universidad (Entrevistado 2).

No sabía qué era la RS, no sabía nada, nada (Entrevistada 1).

No sabía que la gestión educativa tenía una dimensión social (Entrevistada 2).

Yo creía que la RS, sólo era cumplir con lo que me pide mi desempeño laboral asociado a mi cargo (Entrevistado 4).

En lo teórico, las personas refieren haber revisado en la asignatura, teorías asociadas a la responsabilidad desde la filosofía y a la RS en lo particular desde los contextos educativo y organizacional, que previamente no conocían ni siquiera remotamente y que generó en ellas, una ampliación de su comprensión de la RS, identificando variables internas y externas de gestión universitaria, diferentes actores (directivos, académicos, estudiantes, socios comunitarios) y diversos escenarios involucrados (aulas, organizaciones, comunidades locales). Algunos aspectos teóricos que significan como importantes dicen relación con la consideración de la responsabilidad como una condición dialógica propia de los seres humanos; el entendimiento de la RS como gerencia ética e inteligente de los impactos de la universidad y la comprensión de que la RS es tanto una variable interna y externa de gestión y, por tanto, no sólo una condición humana, sino también organizacional.

Me gustaron mucho los autores de Responsabilidad que revisamos, después de las clases me puse a revisar toda la obra de los filósofos Apel y Jonas, el primero porque como decía la profe, recupera la capacidad dialógica de los hombres y Jonas porque con su ética del futuro nos obliga a cuestionarnos por los impactos de nuestras acciones en los otros... me volví fanático de Jonas (Entrevistado 3).

Tuvimos que leer mucho y aprender a discriminar desde qué enfoque de RS sustentaríamos nuestras acciones... A mí el tema de la consideración de los impactos de mis acciones en los otros me pareció muy relevante, porque la RS es un sistema de gestión de los impactos potenciales y reales en los otros... y esos otros pueden ser personas, los alumnos, la comunidad (Entrevistada 3). 
La comprensión conceptual y teórica alcanzada durante la asignatura y posterior a ella, facilitó en las personas, la expresión de cambios en sus prácticas discursivas que fueron reflejando cambios en sus prácticas laborales, ya sea pedagógicas como de gestión y la ruptura de algunos mitos sobre la RS, lo que se evidencia en algunos testimonios.

Yo creía que la RS era tema sólo de las carreras de ayuda, como el trabajo social, la enfermería, nunca imaginé que, en mi cargo como directivo de finanzas, debía también considerarla... Ahora comprendo que lo que hago también impacta en los demás y me doy cuenta que desde mi gestión puedo incorporar acciones de RS... estoy motivado en hacer un proyecto de sala cuna para los hijos de las funcionarias y las estudiantes, así se conocen e integra más la universidad (Entrevistado 5).

Yo creía que la RS sólo debía formarse en los estudiantes, no en nosotros como profesores y que sólo era una competencia general, después me di cuenta que yo, en las asignaturas de carrera, tengo que aportar a su formación en los alumnos (Entrevistada 5).

A continuación, se presenta la Tabla 2. que establece una síntesis conceptual y teórica de la comprensión de la RS en las y los participantes, antes y después de la asignatura, que es posible interpretar desde los significados que le atribuyeron.

Tabla 2. Síntesis conceptual y teórica de la RS.

\begin{tabular}{cc}
\hline Antes & Después \\
\hline RS como beneficencia y filantropía & RS como capacidad y obligación \\
\hline RS individual & RS colectiva \\
\hline RS vertical y asistencial & RS horizontal y recíproca \\
\hline RS de los otros & RS dialógica y consensual \\
\hline RS monológica y abstracta & Responsabilidad social \\
\hline Responsabilidad personal &
\end{tabular}

Fuente: Elaboración propia.

La comprensión de la RS que alcanzaron las personas investigadas, desde los sustentos teóricos y conceptuales, implicó una ruptura de concepciones parciales, más bien centradas en acciones individuales, benéficas y filantrópicas a un entendimiento que la reconoce como capacidad y obligación de responder por las acciones (Sáez, 2001), en que las capacidades individuales y colectivas se disponen para colaborar con la eliminación de obstáculos estructurales, sociales y económicos que impiden la igualdad de oportunidades (Urzúa, 2001). Ello significó que visualizaran su rol formador con mayor amplitud, más allá del aula, de la disciplina que enseñan y de los compromisos de sus cargos, cuando descubrieron la dimensión social de la responsabilidad y, por tanto, de su rol como académicos, que debe también desplegarse en lo colectivo. De allí lograron ser capaces de identificar que muchas de sus acciones de vinculación con el medio antes de la asignatura, eran asistencialistas, unidireccionales y esporádicas, lo que no favorecía la reciprocidad ni horizontalidad que busca la responsabilidad social, restando oportunidad a la expresión de las distintas opiniones y posiciones de enunciación de las personas y/o comunidades con las que se vinculaban, como tampoco la búsqueda dialógica de soluciones a 
los problemas sentidos (Apel, 1985). Por lo mismo, en ocasiones dichas acciones no se mantenían en el tiempo, ni evaluaban sus efectos e impactos presentes ni futuros (Jonas, 1995).

El descubrimiento de que la RS implica a todas las profesiones fue quizás una de las significaciones más importantes alcanzadas por el grupo investigado, puesto que el mito que atribuye RS a profesiones de trato directo con personas o profesiones feminizadas estaba muy enraizado. La progresión de la creencia de que la RS es de otros, a comprenderla desde la perspectiva de la corresponsabilidad solidaria y que implica a todas las disciplinas, contribuyó a que advirtieran que desde la cooperación interdisciplinaria (Apel, 1985) es posible resolver problemáticas que son comunes a las distintas profesiones y que hasta ahora resolvían de manera fragmentada desde sus saberes disciplinares. Esto resulta importante de destacar puesto que como se verá en los análisis siguientes, dio sustento a las articulaciones interdisciplinarias que cristalizaron en la tercera competencia a generar en la asignatura.

La perspectiva monológica centrada en valores abstractos que asociaban a la RS, tales como buenas intenciones o la conciencia de haber cumplido el deber encomendado a sus cargos, fue evolucionando a una comprensión que explicita dichos valores en el engranaje de las relaciones sociales y del interés por las necesidades de los otros. Pero no desde una perspectiva mesiánica, sino desde el reconocimiento de la importancia del diálogo, de escuchar las argumentaciones del otro y de la importancia de llegar al consenso. Aun cuando reconocieron que muchas veces, desde ellos y desde los contextos organizacionales, existen dificultades para aproximarse a transformar sus unidades académicas en comunidades ideales de comunicación (Rubio, 2012), debido a la escasez de prácticas dialógicas, por la tendencia a la toma de decisiones inmediatistas y centralizadas, que estiman son muy propias de las instituciones de educación superior (IES) chilenas por estar enfocadas en el logro de indicadores de gestión más que en generar culturas de RS.

En síntesis, se aprecia un tránsito que evoluciona desde una apreciación individual a una social de la responsabilidad, donde las personas investigadas lograron aprehender que ésta debe afirmarse desde una ética dialógica, histórica y situada, donde la formación profesional que adquieren los estudiantes no se encapsule en lo disciplinar, sino que avance en el reconocimiento de su rol social. Es decir, de cómo es posible formar tanto expertos como ciudadanos, comprometidos con las necesidades de las sociedades en que desplegarán sus saberes específicos, en articulación, con el diálogo y consenso con otras disciplinas y con las personas a quienes dirigen su actuación profesional.

\section{Valoración de la RS como eje de gestión universitaria}

Una segunda capacidad a lograr en la asignatura es valorar la RS como eje central en la gestión de los diferentes sistemas que conforman la educación superior en Chile, especialmente en lo que se refiere a Vinculación con el Medio. En este sentido los contenidos programáticos llevan a las y los estudiantes a revisar las características actuales del sistema de educación superior chileno y de algunos países de Latinoamérica, a discutir las diferencias de las tendencias de proyección social y RS, enfatizando en el rol social que le cabe a la educación superior como fin originario y no sólo la formación profesional para la empleabilidad individual. 
Las personas demuestran cambios cognitivos en lo conceptual y teórico, pero al mismo tiempo expresan cambios actitudinales en su valoración, siendo capaces de otorgar una significación positiva de la RS como eje de gestión. En sus relatos se aprecia que ya no es vista como algo más que hacer o algo que compete a otros, o que resta espacio a sus funciones habituales, o que sólo compete a lo que caracterizan como mal denominadas carreras humanistas, sino que implica a todas las disciplinas.

La asignatura fue un gran aporte para las labores académicas que realizo en el área de diseño. Aprendí la diferencia entre actividades residuales y actividades de Responsabilidad Social que debe llevar a cabo la universidad (Entrevistada 3).

El paso de una concepción inconclusa de RS, marcada por una comprensión residual a que puede reducirse el concepto desde algunos imaginarios que erróneamente la significan desde la filantropía y beneficencia, a una comprensión integral y ciudadana que establece relaciones de reciprocidad, luego de revisar conceptos y teorías, es para las personas investigadas un gran avance en el entendimiento de cómo la significan después de la asignatura. Lo integral para las y los entrevistados alude a un entendimiento sistémico de la RS en que confluyen variables internas y externas de gestión. La valoración positiva se aprecia tanto en lo cognitivo como también en el desarrollo de nuevas habilidades que despliegan en el contexto laboral y educativo, incorporando de manera concreta la RS en su gestión, con acciones mantenidas en el tiempo en lo que se refiere a extensión académica y vinculación con el medio.

Después de la tesis en RS se gestionó una alianza de trabajo con Servicio País, para poder tener una continuidad en el tiempo de las acciones que desarrollamos. Hemos sido un aporte a la comunidad y a la fundación. Ya estamos generando nuevos proyectos, con el fin de generar responsabilidad social para la carrera, institución, docentes y estudiantes. El año pasado realizamos capacitaciones a artesanas mapuches sobre marca e identidad y este año realizamos la Marca para su cooperativa, quedamos a disposición de ellos para seguir apoyándolos (Entrevistada 2).

En complemento, son capaces de otorgarle una significación a su formación en RS como una competencia que aporta tanto a su desarrollo personal como profesional, como también de reconocer el rol social que le compete a la universidad, incorporando en el análisis del estado actual de la educación superior, variables de carácter estructurales, organizacionales e individuales que relevan la diferencia entre RS y el antiguo concepto de proyección social en que se entendía que la universidad de acuerdo a su voluntad y unidireccionalmente le entregaba a la comunidad lo que sobraba de su gestión.

Cuando regresé a mi país, después de estudiar la maestría en Chile me nombraron de inmediato en un cargo directivo vinculado a la RS, aquí me consideran una experta en RS. La asignatura y la tesis en RS me ayudaron a madurar mucho respecto de mi desarrollo personal y mi desempeño profesional. Actualmente represento a mi universidad en reuniones de 
investigación interuniversitarias, pues la región de Gaza-Macia está buscando una unión de todas las instituciones de educación superior para que se conozcan y juntos puedan tener más logros que favorezcan esta región. Eso es Responsabilidad Social. Soy la única mujer representante de mi sede Gaza-Macia, en Mozambique, África (Entrevistada 1).

Una significación necesaria de ilustrar es aquella en que los y las entrevistadas comprendieron, por una parte, académicos y académicas, que su formación en RS, puede y debe exceder el ámbito del aula concretizándose por ejemplo en acciones de aprendizaje servicio como estrategia de RS en que todos las y los estudiantes de una asignatura se vinculen y aporten al desarrollo de un grupo externo (socio comunitario), y, por otra parte, los directivos de áreas de gestión, que su labor inicialmente sustentada desde un enfoque de responsabilidad individual es parte del engranaje de la RS significada como eje de gestión organizacional, por tanto, su quehacer necesariamente impacta en el resto de la organización.

Me ha sido posible enfocar las acciones de VCM de mi área -en forma concretaa la resolución de problemas de la comunidad regional, es decir, con enfoque de RS, teniendo claro los lineamientos y/o directrices necesarias de considerar al momento de su ejecución (Entrevistado 1).

Fue importante esta asignatura en la formación del magíster, los compañeros de los cursos anteriores decían que nos íbamos a enamorar de la RS, porque les cambió la forma de entender la educación, la amplió a una mirada integral (Entrevistada 4).

A modo de síntesis de la valoración de la RS como eje de gestión universitaria que expresan las personas investigadas, se expone la Tabla 3.

La valoración de la RS como eje de gestión universitaria, se caracterizó por una comprensión que la incorpora en todos los ámbitos de acción de un plan estratégico, denotando que las y los académicos lograron deconstruir la perspectiva residual que en general ubica la RS en las instituciones de educación superior, en áreas complementarias a la formación profesional, tales como actividades extra programáticas, asuntos estudiantiles o extensión académica. De esta forma, desde la conceptualización de RSU (Proyecto UCP, 2006), fueron capaces de identificar que debe y puede estar incorporada transversalmente en la docencia, la investigación, la gestión y la extensión académica.

La consideración de que la RS es sólo una variable externa en que la universidad se vincula con el entorno, generalmente a través de actuaciones unilaterales y aisladas de voluntariado, fue destronada cuando desde la propuesta de Vallaeys (2006) lograron identificar que la universidad tiene tanto responsabilidad con el personal que labora en ella y con los estudiantes que forma (RS interna), como con el entorno con que se vincula (RS externa). Esto significó un cambio paradigmático en su comprensión de lo que hasta ahora habían significado como RS, porque los llevó a descubrir que la gestión de esta no es sólo externa, sino que debe considerar los entornos interno y externo, desde actuaciones bidireccionales y sostenidas en el tiempo. 
Tabla 3. Síntesis de la valoración de la RS como eje de gestión universitaria.

\begin{tabular}{|c|c|}
\hline Antes & Después \\
\hline $\begin{array}{l}\text { RS residual incorporada en una unidad de } \\
\text { gestión universitaria }\end{array}$ & $\begin{array}{c}\text { RS integral incorporada en todos los ámbitos } \\
\text { del plan estratégico }\end{array}$ \\
\hline RS como una variable de gestión externa & $\begin{array}{l}\text { RS conformada por variables internas y } \\
\text { externas de gestión }\end{array}$ \\
\hline $\begin{array}{l}\text { Visión fragmentada de los impactos de } \\
\text { gestión universitaria }\end{array}$ & $\begin{array}{l}\text { Visión sistémica y multifactorial de los } \\
\text { impactos de gestión universitaria }\end{array}$ \\
\hline $\begin{array}{l}\text { RS incorporada en unidad de extensión } \\
\text { académica como actividad extra programática }\end{array}$ & $\begin{array}{l}\text { RS incorporada transversalmente en gestión, } \\
\text { docencia, investigación y extensión académica }\end{array}$ \\
\hline $\begin{array}{l}\text { RS como proyección social, la universidad } \\
\text { otorga lo que le sobra a la comunidad }\end{array}$ & $\begin{array}{l}\text { RS como eje fundamental del rol social que le } \\
\text { corresponde a la educación superior }\end{array}$ \\
\hline RS como actividades de asistencialismo & $\begin{array}{l}\text { RS concretizada en acciones de } \\
\text { aprendizaje servicio }\end{array}$ \\
\hline
\end{tabular}

Fuente: Elaboración propia.

Esto se complementa con el aprendizaje de los impactos de la gestión universitaria, desde una perspectiva sistémica y multifactorial, donde el modelo de Vallaeys colaboró en que distinguieran que la RS implica ocuparse por los impactos que las acciones humanas y organizacionales generan en los otros y en el entorno. Resulta importante especificar que, en una primera instancia, aunque de manera parcial, sólo fueron capaces de identificar los impactos educativos y sociales de la gestión de las IES. Sin embargo, los impactos organizacional, científico y tecnológico, fueron más difíciles de detectar debido a que su comprensión inicial de la RS, la apreciaban alejada de dichos impactos. Esto ocurrió porque en general las acciones de RS que conocían estaban vinculadas en primer lugar a lo social y en segunda instancia a lo educativo. Así reconocieron que la universidad no sólo forma profesionales, sino que es también un referente y actor social de relevancia, que con la orientación que imprime en los planes de estudio influye en la deontología y ética profesional, tanto desde su comportamiento organizacional como desde sus elecciones epistemológicas.

Esto les llevó a darse cuenta que las instituciones de educación superior no pueden continuar ancladas al antiguo concepto de proyección social, sino que deben implicarse colaborativamente con los entornos cercanos y remotos para recuperar el rol social que les cabe por definición, evitando transmitir a sus estudiantes y a la sociedad, que su accionar se reduce a la colocación de jóvenes en el mercado. Esto fue altamente valorado por los participantes y de fácil comprensión, debido a que lo relacionaron con las acciones de vinculación con el medio que la universidad a que pertenecen efectúa en las catorce regiones del país en que tiene presencia, lo que además expresaron es una exigencia de los planes de desarrollo estratégicos, locales y nacional, en que se espera que exista desde las distintas unidades académicas, relacionamientos con las comunidades locales.

Como corolario lograron distinguir la relevancia que adquieren hoy las acciones de Aprendizaje Servicio que, como estrategia sustentada desde la RS, señalaron, aporta tanto a la 
formación de actitudes prosociales en los estudiantes universitarios como fundamentalmente a la solución de las necesidades detectadas de los socios comunitarios, desde actuaciones profesionales reflexivas y co-construidas con ellos. Así lograron diferenciarla de actuaciones asistenciales o de voluntariado profesional y no profesional que habían aplicado unilateral y esporádicamente, hasta antes de la asignatura.

\section{Desarrollo de habilidades para la formulación de proyectos de VCM con RS}

La tercera capacidad que se espera lograr en la asignatura hace referencia a desarrollar habilidades que favorezcan la formulación y puesta en marcha de proyectos de VCM con estándares de RS, en las distintas unidades en que se desempeñan quienes la cursan: docencia, investigación, gestión y/o extensión académica. De esta forma se pretende transversalizar, desde la comprensión conceptual y teórica hacia el actuar profesional, la valoración de que la RS es una variable de gestión fundamental de los sistemas educativos modernos. Para ello fue necesario, como lo retratan los testimonios, avanzar desde una comprensión incipiente e incompleta a una global y completa en todos los ámbitos que abarca la RS, donde las personas investigadas desplegaron capacidades de pensamiento crítico y reflexivo para efectuar análisis comparados de experiencias universitarias en RS de Chile, Latinoamérica y Europa, como también demostraron capacidades transferenciales para proponer políticas de RS vinculadas a sus áreas de gestión y formular proyectos de VCM con estándares de RS.

Fueron difíciles algunos trabajos, a mí me costó mucho hacer el ensayo individual, porque no sabía nada de RS y lo que sabía, con la asignatura comprendí que estaba equivocado... pero era necesario porque me motivó para el trabajo final, para hacerlo con compañeros de otras carreras y otras sedes... jimagínate! (Entrevistado 3).

Yo me motivé tanto con ese trabajo de hacer políticas de RS y un proyecto de RS que hasta hice la tesis del magíster en RS y postulé a un fondo concursable para financiar la idea de hacer un centro de atención integral en la sede, donde aporten todas las carreras (Entrevistado 4).

Para profundizar en el análisis de esta capacidad e indagar en cómo las dos anteriores tributan a ella, durante las entrevistas, se consultó a las personas investigadas respecto de los contenidos, actividades y evaluaciones desarrolladas en las unidades del programa de la asignatura, en el entendido de que esta capacidad daría evidencia de nuevas acciones o prácticas sociales en las y los entrevistados, vinculadas a sus nuevas significaciones de RS. Con especial énfasis se examinaron y analizaron los reportes de los contenidos del trabajo final que sintetizan las tres capacidades a lograr mediante la formulación de proyectos de VCM con estándares de RS, donde se espera que se cumpla el requisito de articulación de diferentes disciplinas, diversas áreas de gestión y estamentos educativos en diferentes sedes de la institución investigada. Siguiendo lo planteado por Fernández (2015), se aprecia que la tendencia de integración que sobresale 
es la interdisciplinar, seguida por la multidisciplinar. Es decir, la mayoría de los proyectos consideran reciprocidad de intercambios interdisciplinares, seguidos por proyectos donde varias disciplinas aportan a una misma situación desde la multidisciplina, sin implicar modificación ni enriquecimiento entre disciplinas, como se aprecia en la Tabla 4. Proyectos de RS con estándares de VCM.

En igual sentido, durante las entrevistas, fueron exploradas las temáticas abordadas por las tesis para graduarse del programa de magíster que se centraron en temáticas de RS o afines, donde cabe mencionar que dos tesis profundizaron y desarrollaron las ideas propuestas previamente en el trabajo final de la asignatura de VCM y RS, lo que denota también capacidades para incorporar la RS en la gestión estratégica de las unidades a cargo. Esto puede apreciarse en la Tabla 5. Tesis de Magíster en Gestión en Educación Superior, asociadas a temáticas de RS.

Esta revisión implicó analizar los contenidos tratados en la asignatura, aplicados por los estudiantes en la fundamentación de sus proyectos de VCM con RS y en las tesis en RS, con el fin de identificar aquellos conocimientos que para ellos fueron comprendidos

Tabla 4. Proyectos de VCM con estándares de RS.

\begin{tabular}{|c|c|c|}
\hline Proyecto de VCM con RS & Disciplinas y unidades integradas & Nivel de integración \\
\hline $\begin{array}{l}\text { Identificación de prácticas culturales y } \\
\text { gastronómicas de la Araucanía para potenciar } \\
\text { su valorización patrimonial y turística }\end{array}$ & $\begin{array}{l}\text { Diseño, Instituto Profesional, zona sur. } \\
\text { Gastronomía, Centro de Formación Técnica, } \\
\text { zona sur. }\end{array}$ & Interdisciplinar \\
\hline $\begin{array}{l}\text { Programa de formación en competencias } \\
\text { parentales, orientado al buen trato y } \\
\text { crianza responsable de niños y niñas de } \\
\text { establecimientos educacionales vulnerables }\end{array}$ & $\begin{array}{l}\text { Psicología, Universidad, zona norte. } \\
\text { Derecho, Universidad, zona sur. }\end{array}$ & Interdisciplinar \\
\hline $\begin{array}{l}\text { Atención biopsicosocial en salud para } \\
\text { familias de caleta de pescadores, basada en } \\
\text { diagnóstico participativo de necesidades } \\
\text { e implementación de intervenciones } \\
\text { interdisciplinares }\end{array}$ & $\begin{array}{l}\text { Nutrición y Dietética Psicología, Educación } \\
\text { Derecho, } \\
\text { Universidad zona norte. }\end{array}$ & Interdisciplinar \\
\hline $\begin{array}{l}\text { Implementación de guardería infantil gratuita } \\
\text { para estudiantes, funcionarios y comunidad } \\
\text { externa, con miras a disminuir índices de } \\
\text { deserción estudiantil y ausentismo laboral }\end{array}$ & $\begin{array}{l}\text { Educación Parvularia, Universidad, zona } \\
\text { norte. } \\
\text { Dirección de Finanzas, Centro de Formación } \\
\text { Técnica, Instituto Profesional, Universidad, } \\
\text { zona norte }\end{array}$ & Multidisciplinar \\
\hline $\begin{array}{l}\text { Implementación de centro de atención } \\
\text { profesional integral para estudiantes y } \\
\text { comunidad en localidad zona sur }\end{array}$ & $\begin{array}{l}\text { Nutrición y Dietética } \\
\text { Kinesiología, Enfermería } \\
\text { Fonoaudiología } \\
\text { Psicología, Derecho } \\
\text { Medicina Veterinaria, Universidad zona sur. } \\
\text { Servicio Social, Construcción, Preparador } \\
\text { Físico, Instituto Profesional, zona sur. } \\
\text { Podología, Administración, Centro de } \\
\text { Formación Técnica, zona sur. }\end{array}$ & Multidisciplinar \\
\hline
\end{tabular}

Fuente: Elaboración propia. 
Tabla 5. Tesis de Magíster en Gestión en Educación Superior, asociadas a temáticas de RS.

\begin{tabular}{lc}
\hline \multicolumn{1}{c}{ Título de la tesis } & Profesionales a cargo \\
\hline $\begin{array}{l}\text { Sistematización de prácticas culturales y gastronómicas de la } \\
\text { Araucanía desde el enfoque de la RS Territorial }\end{array}$ & $\begin{array}{c}\text { Diseñadora } \\
\text { Chef }\end{array}$ \\
\hline $\begin{array}{l}\text { Análisis de prácticas de vinculación con el medio que son evidencia } \\
\text { de RS en una universidad de zona centro }\end{array}$ & Profesora de Filosofía \\
\hline $\begin{array}{l}\text { Implementación de un centro de atención integral con estándares de } \\
\text { RS desde una universidad del sur }\end{array}$ & $\begin{array}{c}\text { Biólogo Marino } \\
\text { Ingeniero en Informática }\end{array}$ \\
\hline $\begin{array}{l}\text { Actitud identitaria en estudiantes, respecto al sello institucional, } \\
\text { como indicador de gestión universitaria en zona norte }\end{array}$ & Profesor de Religión \\
\hline
\end{tabular}

Fuente: Elaboración propia.

como aprendizajes significativos. Esto es lo que se representa en la Fig. 1. Aprendizajes Significativos de RS que reúne las tendencias más frecuentes, diferenciadas por niveles de importancia.

El mayor nivel de importancia se otorgó a tres conceptos, presentes en la mayoría de las propuestas revisadas, coincidiendo en relevar como ejes principales: la RS como sustento teórico conceptual de las propuestas, la estrategia de aprendizaje servicio como herramienta para concretizarla y la necesidad de la gestión estratégica de la RS en los planes de desarrollo de las diversas unidades desde donde se formularon los proyectos.

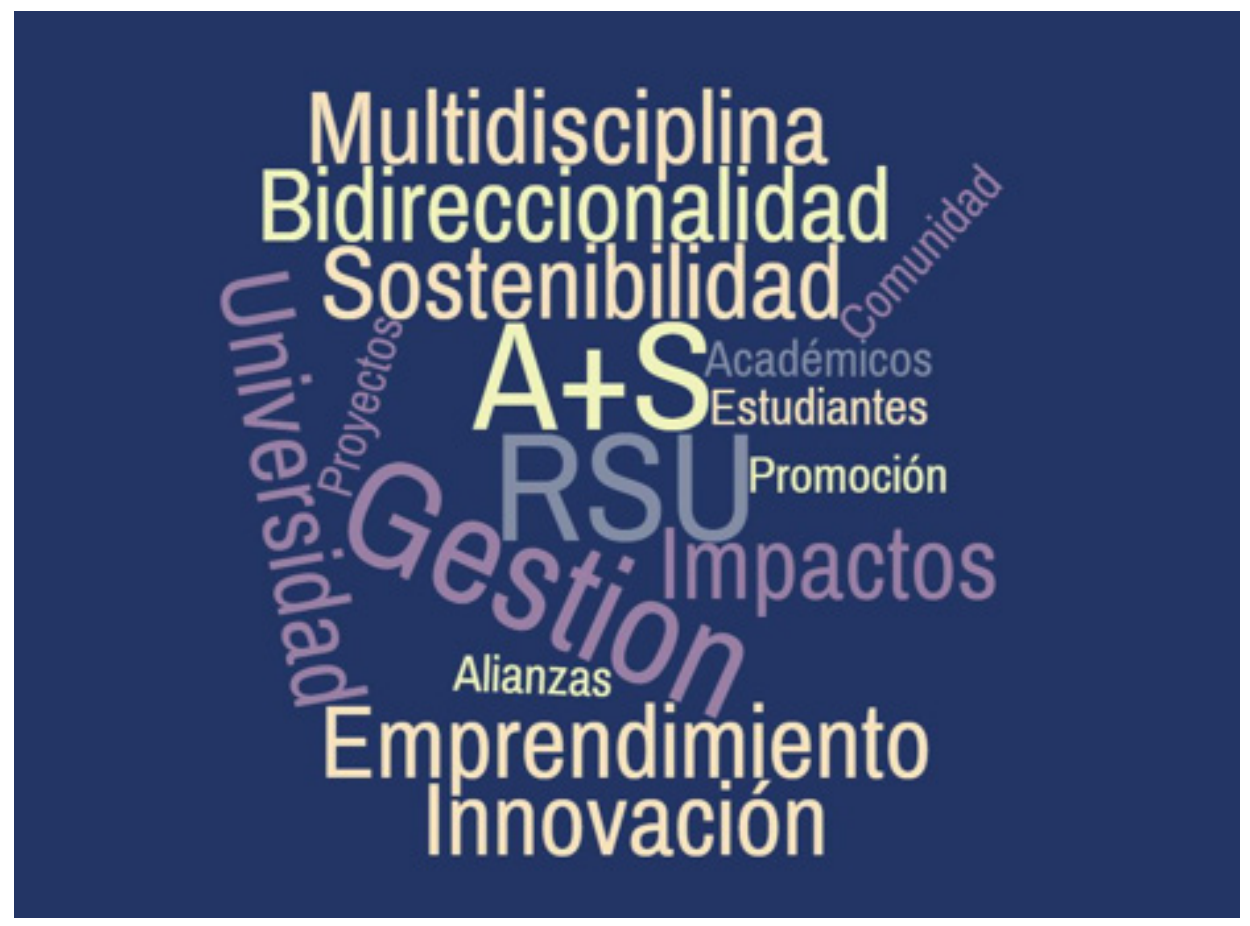

Fig. 1. Aprendizajes significativos de RS.

Fuente: Elaboración propia. 
En segundo lugar, la multidisciplina (distintas carreras, distintas sedes, distintos estamentos, aportando en la solución de una misma situación), bidireccionalidad (relaciones horizontales y recíprocas entre estos diferentes actores) y la sostenibilidad (estrategias mantenidas en el tiempo), son características presentes en las propuestas, lo que demuestra la ruptura de la visión fragmentada disciplinar, al lograr darse cuenta de la necesidad de la cooperación interdisciplinar y de cómo ésta debe avanzar a niveles de mayor integración y diálogo, como la interdisciplina e idealmente la transdisciplina. En igual nivel de importancia aparecen los conceptos de emprendimiento e innovación, lo que es relevante de analizar porque las y los académicos lograron aplicar en sus proyectos la relación de estos conceptos con la RS, que hoy desafía a las IES a situarse desde estos modernos ejes de gestión que implican una nueva forma de gestionar la formación profesional de las generaciones actuales y futuras, junto con aportar al desarrollo social de comunidades, organizaciones, territorios. Es decir, formando profesionales y emprendedores que formen emprendedores por medio de la innovación social. Posteriormente se aprecian los conceptos de universidad e impactos (ocupación por potenciales efectos de la propuesta) como elementos clave presentes en los proyectos, lo que denota que comprendieron como un requisito indispensable que las IES deben ocuparse por los potenciales efectos de su gestión en los entornos en que se ubica.

Finalmente se identifican los conceptos asociados a los actores que consideraron en sus propuestas, otorgándoles un mismo nivel de relevancia a académicos, estudiantes, comunidad, sin atribuir relaciones de dependencia, estableciendo alianzas para la formulación de proyectos centrados, en general, en la promoción. Es interesante destacar que no se apreció formulación de proyectos orientados a la rehabilitación, recuperación, mejoramiento, reparación, sino en general son proyectos que se dirigen a la organización entre personas o comunidades, en el entendido de que la RS logró ser comprendida por las personas investigadas como un valor, una capacidad, una conducta o actitud pro social y no como una acción asistencialista vertical desde un tercero hacia un otro, rompiendo con la significación de proteger a los más débiles o de reemplazarlos en el desarrollo de habilidades, entregándoles recursos o beneficios asistenciales.

\section{CONCLUSIONES}

A partir de los resultados presentados es dable concluir respecto de los significados que las personas atribuyen a la RS luego de haber cursado la cátedra Vinculación con el Medio y Responsabilidad Social en Educación Superior, con miras a responder al propósito de este estudio. En las tres versiones en que se ha dictado esta cátedra, se ha orientado a la formación de treinta y ocho académicas, académicos y/o directivos de las más diversas disciplinas (Salud, Cs. Sociales, Jurídicas, Educación) y áreas de gestión (Docencia, Administración, Finanzas, Formación Valórica) de catorce sedes de una universidad en Chile y de tres académicas de su universidad homóloga en Mozambique, África, evidenciando un proceso de deconstrucción en las competencias del saber (conocimientos), saber hacer (habilidades) y saber ser (valores y actitudes), en lo que a RS se trata. 
Los y las participantes dan cuenta de un cambio notable en lo conceptual desde un polo inicial de nulo o casi nulo conocimiento de RS, en general teñido por mitos y acepciones incompletas, hasta alcanzar significaciones con apreciaciones diversas y actualizadas de acuerdo con los ámbitos de gestión en que ésta puede ubicarse. De esta forma lograron comprenderla como una capacidad humana que obliga individualmente a responder ante la sociedad, pero también avanzaron hacia comprensiones más complejas, reconociendo que es también una capacidad exigible al Estado, a la administración pública, a los gobiernos, a las organizaciones y especialmente a la educación superior, para responder ante la propia universidad (RS interna), sus estudiantes y la comunidad en que se inserta (RS externa). De esta manera identifican los procesos clave en que puede y debe incorporarse la RSU: gestión, docencia, investigación y extensión. Este fue para ellos uno de los principales aprendizajes que les motivó a incorporarla en la gestión estratégica de las unidades a su cargo, pasando de considerarla sólo como una actividad asistencialista, de otras áreas o unidades, a comprenderla como un nuevo paradigma de gestión que debe estar incorporado en su quehacer, ya sea desde la docencia, la investigación o la dirección de sistemas educativos, para asegurar que las instituciones de educación superior cumplan con el rol social que les corresponde por esencia. Por tanto, se aprecia en las y los participantes un cambio en la comprensión de la educación desde concebirla como formadora de especialistas orientada a la empleabilidad individual, hasta entenderla como formación de ciudadanos socialmente responsables, implicados con sus entornos cercanos y remotos.

La reflexión teórica a que llegan las personas investigadas es necesaria de rescatar cuando identifican los impactos y los tránsitos que pueden seguir las universidades para aproximarse a la RS. Con relación a los impactos, cuestionan los tributos y daños que puede generar la educación superior en la formación de las nuevas generaciones cuando no se conecta adecuadamente con el medio en que se desenvolverán laboralmente y limita la formación a la experticia propia y fragmentada de cada disciplina sin incorporar la tributación que cada profesional puede otorgar al desarrollo local y social (impacto educativo y epistemológico), desde perspectivas multi, inter y transdisciplinares de ser posible. Relevan aquí la adecuada implementación de acciones planificadas de aprendizaje servicio durante la formación de pregrado como estrategia contemporánea para concretizar la RS que contribuya tanto a generar compromiso social, actitudes prosociales y comportamientos socialmente responsables en los estudiantes y académicos, en los territorios y comunidades con que se vincula e inserta la universidad.

Un hallazgo muy relevante para este estudio y para las personas investigadas fue la consideración de la RS más allá de la formación en los estudiantes de pregrado, comprendiendo que parte de los impactos organizacional y social de las instituciones de educación superior está en la gestión que ellos y ellas desarrollen y, por tanto, no puede reducirse a la formación de competencias en los estudiantes ni a la puesta en marcha de actividades de extensión en ocasiones desconectadas de los proyectos educativos de las IES y de los perfiles de egreso de distintas disciplinas. Esto fue comprendido como una perspectiva incompleta de RS, por ello critican que las universidades no se ocupen de gestionar los impactos organizacionales en los campos laboral y 
medioambiental especialmente y los impactos sociales cuando se dictan carreras cuyos campos ocupacionales están saturados generando cesantes ilustrados, precarizando sus condiciones de inserción y mantención en el campo laboral.

En relación con los tránsitos respecto de los que puede avanzar una institución de educación superior para aproximarse a estándares de RS, reconocen que la capacidad dialógica y la visión sistémica no están aún instaladas en todos los sistemas de educación superior, por eso la RS se encapsula en general en formación de pregrado y extensión académica, desde una visión residual, externa y asistencialista, más cercana al marketing que a la formación ciudadana. Esto es coincidente con lo señalado en la CRES 2018 donde se declara que el limitado enfoque de proyección social y extensión universitaria visualiza a la RS como apéndice de la función central de formación estudiantil y producción de conocimientos. En este sentido identifican y critican las tensiones a las que se enfrentan las instituciones de educación superior que, desde el modelo neoliberal, las obligan a cumplir con estándares de acreditación e indicadores de crecimiento, no así de desarrollo ni de formación ciudadana, supeditando otras áreas, consideradas por ellos más relevantes, como lo es la calidad de la formación de las y los jóvenes profesionales. Sin embargo, están ciertos de que si la RS fuese considerada como un eje de gestión transversal presente como valor y variable de acción de los planes estratégicos, se podría avanzar en que las IES se transformen en comunidades ideales de comunicación, aportando tanto a la empleabilidad individual, como al desarrollo social y económico de la sociedad en que se insertan.

En síntesis, es posible detentar que la formación de profesionales y ciudadanos lograría asegurarse en parte, desde la formación de directivos y académicos socialmente responsables. Por ello, la declaración de ser universidades socialmente responsables se transforma en práctica cuando en la gestión, la docencia, la investigación y la extensión, hay reconocimiento del rol social que le corresponde a la universidad, considerando como eje estratégico a la RS, como valor de todos los actores involucrados: académicos, directivos, funcionarios y estudiantes, deconstruyendo el antiguo paradigma asistencialista, para comprenderla como práctica dialógica y de reciprocidad permanente, lo que sólo se logrará cuando se reconozca a la educación como derecho humano fundamental y se incorpore a la RS como eje estratégico de todo el quehacer de las instituciones de educación superior, sin supeditarla a un solo ámbito de gestión, a una sola disciplina y a un solo actor de las comunidades educativas, superando como lo establece la CRES 2018, la visión disciplinaria y catedrática de la educación para vincularse con la sociedad para crear y diseñar conjuntamente nuevos escenarios de generación democrática de conocimiento.

\section{AGRADECIMIENTOS}

Se agradece al Fondo de Investigación de la Universidad a la que pertenece la Investigadora Responsable por el apoyo para la realización de este estudio que es parte del proyecto de investigación regular 2019-2020, código 039942. 


\section{REFERENCIAS}

Apel, K. (1985). La transformación de la filosofía. Madrid: Taurus.

Apel, K. (2007). La Globalización y una ética de la responsabilidad: reflexiones filosóficas acerca de la globalización. Buenos Aires: Ediciones Prometeo Libros.

Bacigalupo, L. (2006). Una aproximación desde los conceptos de Sociedad Civil y Responsabilidad Social Universitaria, Universidad y Desarrollo Regional. Santiago: Consorcio de Universidades.

Canales, M. (2006). Metodologías de Investigación Social: Introducción a los Oficios. Santiago: Ediciones Lom.

Cañón, O., Peláez, M., \& Noreña, N. (2005). Reflexiones sobre el socioconstruccionismo en psicología. Diversitas: Perspectivas en Psicología, 1(2), 238-245. http://doi.org/10.15332/ s1794-9998.2005.0002.11

Conferencia Regional de Educación Superior (CRES). (2018). Declaración de la Tercera Conferencia Regional de Educación Superior-2018. Córdoba: CRES.

Fernández, A. (2015). La práctica de la RSU con y para estudiantes: aportes al fortalecimiento del vínculo entre la universidad y sociedad. En I. Fernández et al. (Eds.), Experiencias Iberoamericanas en responsabilidad social universitaria (pp. 157-193). Medellín: FunLam.

Flick, U. (2012). Introducción a la investigación cualitativa. Madrid: Ediciones Morata.

Flores, R. (2009). Observando Observadores: Una Introducción a las Técnicas Cualitativas de Investigación Social. Santiago: Ediciones Universidad Católica de Chile.

Gergen, K. (2006). Realidades y Relaciones: Aproximación a la Construcción Social. Barcelona: Editorial Paidós Básica.

Gómez-Mendoza, M. Á. (2000). Análisis de contenido cualitativo y cuantitativo. Definición, clasificación y metodología. Revista de Ciencias Humanas, 20, 129-138. ISSN: 01219677. Disponible en: http://www.utp.edu. co/ chumanas/revistas/revistas/rev20/ gomez.htm

Jonas, H. (1995). El principio de Responsabilidad: Ensayo de una Ética para la civilización Tecnológica. Barcelona: Herder.

Lagares, P., \& Puerto, J. (2001). Población y muestra. Sevilla: Universidad de Sevilla.

Martínez-Salgado, C. (2012). El muestreo en investigación cualitativa. Principios básicos y algunas controversias. Revista Ciencia \& Saúde Coletiva, 17(3), 613-619. http://doi. org/10.1590/S1413-81232012000300006

Mella, O. (2003). Metodología Cualitativa en Ciencias Sociales y Educación. Santiago: Editorial Primus.

Navarro, G., Rubio, V., Lavado, S., \& Minnicelli, A. (2017). Razones y propósitos para incorporar la responsabilidad social en la formación de personas y en organizaciones de Latinoamérica. Revista Latinoamericana de Educación Inclusiva, 11(2), 51-72. http:// doi.org/10.4067/S0718-73782017000200005

Organización de las Naciones Unidas para la Educación, la Ciencia y la Cultura (Unesco). (2009). Conferencia mundial sobre la educación superior-2009: La nueva dinámica de la educación superior y la investigación para el cambio social y el desarrollo. París: 
UNESCO. Última visita 24 de abril de 2018. Recuperado desde www.unesco.org/ education/WCHE2009comunicado_es.

Pakman, M. (1997). Construcciones de la Experiencia Humana. Barcelona: Gedisa.

Pérez, G. (2002). Investigación Cualitativa. Retos e Interrogantes, Tomo II: Técnicas. Madrid: Editorial La Muralla, S.A.

Pizarro, V., \& Hasbún, B. (2019). Aprendizaje Servicio en la Educación Superior Chilena. Santiago: Ediciones CEA-FEN Universidad de Chile.

Preciado, P. (2002). Manifiesto contra-sexual. Madrid: Opera Prima.

Proyecto Universidad Construye País (2002). Seminario La Universidad Construye País. La

Responsabilidad Social de la Universidad de cara al Chile del 2010. Pontificia Universidad

Católica de Valparaíso.

Proyecto Universidad Construye País (2006). Responsabilidad Social Universitaria: Una Manera de ser Universidad. Santiago: Corporación PARTICIPA Y AVINA.

Rubio, V. (2012). La universidad como constructora de ciudadanos socialmente responsables. Santiago: Ediciones Universidad Santo Tomás.

Rubio, V. (2013). Responsabilidad Social en Educación: Apuntes para su comprensión desde la investigación cualitativa. Revista Investigaciones en Educación, 13(2), 19-40.

Rubio, V. (15 de noviembre de 2019). La educación superior en los tiempos del cólera. El Mercurio de Valparaíso. http://www.mercuriovalpo.cl/impresa/2019/11/21/full/ cuerpo-principal/10/

Sabino, C. (1986). El proceso de la investigación social. Buenos Aires: Editorial Humanitas.

Sáez, O. (2001). La responsabilidad social de las universidades: una guía para la discusión. Santiago: Proyecto Universidad Construye País.

Salgado, A. (2007). Investigación cualitativa: diseños, evaluación del rigor metodológico y retos. Liberabit, 13(13), 71-78.

Siqueira, J. (2001). El principio de responsabilidad de Hans Jonas. Acta Bioethica, año 7(2), 277-285. http://dx.doi.org/10.4067/S1726-569X2001000200009

Urzúa, R. (2001). La responsabilidad social de las universidades: una guía para la discusión. Santiago: Proyecto Universidad Construye País.

Vallaeys, F. (2006). Responsabilidad Social Universitaria: de la teoría a la práctica. Lima: Pontificia Universidad Católica del Perú.

Vallaeys, F. (2009). Responsabilidad Social Universitaria: Manual de Primeros Pasos. México D.F.: Mc Graw Hill Interamericana.

Vasilachis de Gialdino, I., Ameigeiras, A., Chernobilsky, L., Giménez, V., Mallimaci, F., Mendizábal, N., Neiman, G., Quaranta, G., \& Sonería, A. (2006). Estrategias de Investigación Cualitativa. Barcelona: Gedisa.

Vivero, L., Molina, W., \& Standen, D. (2020). Un análisis crítico al modelo aprendizaje servicio: su implementación en la Universidad Católica de Temuco. Revista Sophia Austral, 25(1), 121-137. http://dx.doi.org/10.4067/S0719-56052020000100121 